\title{
Tim-4 protects mice against lipopolysaccharide-induced endotoxic shock by suppressing the NF-KB signaling pathway
}

\author{
Liyun Xu ${ }^{1,2,5}$, Peiqing Zhao ${ }^{1,3,5}$, Yong Xu ${ }^{1,5}$, Lishuang Gao ${ }^{4}$, Hongxing Wang ${ }^{1}$, Xiaoxia Jia ${ }^{1}$, Hongxin Ma ${ }^{1}$, \\ Xiaoxong Liang ${ }^{1}$, Chunxong $\mathrm{Ma}^{1}$ and Lifen Gao ${ }^{1}$
}

Endotoxic shock is the primary cause of morbidity and mortality in hospital patients, creating an urgent need to explore the mechanisms involved in sepsis. Our previous studies showed that T-cell immunoglobulin- and mucin-domaincontaining molecule-4 (Tim-4) attenuated the inflammatory response through regulating the functions of macrophages. However, the mechanism by which Tim- 4 does this has not been fully elucidated. In this study, we found that Tim- 4 expression was increased in lipopolysaccharide (LPS)-induced endotoxic shock. Interestingly, the survival rate of mice in the Tim- 4 overexpression group was higher than that of the control group after LPS administration. To investigate the function of Tim-4 in LPS-induced inflammation, we further demonstrated that Tim-4 attenuated LPS-induced endotoxic shock by inhibiting cytokine production by macrophages. Blocking expression of Tim- 4 and nuclear factor-kappa B (NF- $K B$ ) signal inhibition showed that Tim-4 inhibited cytokine production via NF-KB signaling pathway. This study indicates that Tim-4 may exert its immune modulation by regulating inflammatory factor secretion and might act as a novel potential target for inflammatory diseases, especially endotoxic shock.

Laboratory Investigation (2016) 96, 1189-1197; doi:10.1038/labinvest.2016.94; published online 12 September 2016

Endotoxic shock, also referred to as septic shock, is one of the leading causes of morbidity and mortality in hospital patients, ${ }^{1}$ as well as in experimental animal models. ${ }^{2}$ The innate immune system, including innate immune cells such as macrophages and dendritic cells, is the first line of defense against endotoxic shock, which is induced mainly by Gramnegative bacteria through stimulation of Toll-like receptors (TLRs). ${ }^{3}$ TLR4 is engaged by lipopolysaccharide (LPS) from Gram-negative bacteria, whereas other components of microbes are recognized via other TLRs. ${ }^{4}$ However, all TLRs activate nuclear factor-kappa B (NF- $\kappa \mathrm{B})$ and the mitogenactivated protein kinase pathway, and the engagement of TLRs results in a potent inflammatory response characterized by the release of abundant proinflammatory cytokines and chemokines. ${ }^{5}$ In clinical practice, endotoxic shock is defined as a systemic inflammatory response to the presence of a documented infection in critically ill patients. ${ }^{6}$ Although multiple treatments are currently used to deal with severe endotoxic shock, due to the complicated nature of sepsis, endotoxic shock remains as the most common cause of death in intensive care units. ${ }^{7,8}$ Therefore, substantial efforts should be made to clarify the mechanisms of immunopathology involved in endotoxic shock in order to develop new therapeutic strategies.

T-cell immunoglobulin- and mucin-domain-containing molecule-4 (Tim-4) was first discovered in 2001 as a novel immune regulator. ${ }^{9}$ In contrast to other Tim family members, Tim-4 is selectively expressed on antigen-presenting cells, especially on macrophages and mature myeloid-derived dendritic cells. ${ }^{9}$ Tim-4 acts as a ligand for Tim-1, which prompts $\mathrm{T}$-cell expansion and survival. ${ }^{10,11}$ It has been associated with a variety of immune regulatory disorders including allergy, asthma, and autoimmunity. ${ }^{12}$ Moreover, Tim-4 promotes macrophages to engulf the apoptotic or

Department of Immunology, Key Laboratory for Experimental Teratology of Ministry of Education, Shandong Provincial Key Laboratory of Infection \& Immunology, Shandong University School of Medicine, Jinan, Shandong, China; ${ }^{2}$ Cell and Molecular Biology Laboratory, Zhoushan Hospital, Zhoushan, Zhejiang, China; ${ }^{3}$ Department of Central Laboratory, Zibo Central Hospital, Zibo, Shandong, China and ${ }^{4}$ College of Science and Engineering, Shandong University of Traditional Chinese Medicine, Jinan, Shandong, China

Correspondence: Professor L Gao, Department of Immunology, Key Laboratory for Experimental Teratology of Ministry of Education, Shandong Provincial Key Laboratory of Infection \& Immunology, Shandong University School of Medicine, 44 Wenhua Xi Road, Jinan, Shandong 250012, China.

E-mail: glfflg@sdu.edu.cn

${ }^{5}$ These authors contributed equally to this work.

Received 5 February 2016; revised 8 July 2016; accepted 27 July 2016 
necrotic bodies by recognizing phosphatidylserine exposed on the surface of apoptotic cells and is involved in innate immunity-mediated diseases. ${ }^{13-15}$ Our previous data confirm that Tim-4 negatively regulates macrophages and reduces the levels of proinflammatory cytokines stimulated by Concanavalin $\mathrm{A}$ (ConA).${ }^{16}$ It is known that ConA, LPS, or interferon- $\gamma$ stimulation induces Tim- 4 expression in macrophages. ${ }^{16,17}$ Rodriguez-Manzanet $\mathrm{R}$ and colleagues ${ }^{11}$ also reported that the expression of Tim- 4 on $\mathrm{CD} 11 \mathrm{c}^{-} \mathrm{CD} 11 \mathrm{~b}^{+}$splenic macrophages is increased after LPS stimulation, suggesting the upregulation of Tim-4 upon activated macrophages induced by LPS. Moreover, the expression of Tim-4 on DCs phagocytosed Escherichia coli particles increases 3 - to 4 -fold, ${ }^{18}$ indicating that Tim-4 may involve in the development of endotoxic shock. However, the mechanism of Tim-4 in LPS-induced endotoxin shock remains elusive.

In this study, we developed an experimental endotoxinstimulated septic shock model in order to address the role of Tim-4 in the development of endotoxic shock. We found that Tim-4 protected mice from LPS-induced septic shock by inhibiting proinflammatory cytokines in an NF- $\kappa \mathrm{B}$-dependent manner.

\section{MATERIALS AND METHODS}

\section{Animals}

Six to eight-week-old female BALB/c mice were purchased from the Shandong University Laboratory Animal Center (Shandong, China). All mice were housed in the animal facilities under pathogen-free conditions. Experimental procedures were approved by the Animal Care and Use Committee of Shandong University.

\section{Cell and Plasmids}

RAW264.7 cells (ATCC TIB-71) were purchased from Shanghai Institutes for Biological Science (Shanghai, China) and cultured in DMEM media, supplemented with $10 \%$ fetal bovine serum and penicillin/streptomycin. Stable macrophage cell lines overexpressing Tim-4 (RAW264.7-Tim-4) and the control cell line (RAW264.7-pcDNA3) were established in our laboratory. The plasmids pEGFP-N1-Tim-4, mU6Tim-4-shRNA, and pcDNA3-Tim-4 were constructed and conserved.

\section{LPS-Induced Endotoxic Shock}

BALB/c mice were administered LPS (0111:B4) (Sigma, St Louis, MO, USA) at $15 \mathrm{mg} / \mathrm{kg}$ by intraperitoneal injection to induce endotoxic shock, and saline was used as control. Eight hours later, the bone marrow, spleen, lung, and kidney were collected to detect Tim-4 mRNA and protein expression. Subsequently, overexpression of Tim- 4 was conducted in animal models. In detail, the mixture of $60 \mu \mathrm{g}$ pEGFP-N1Tim- 4 or pEGFP-N1 plasmid DNA and PEI at 10:1 of N:P was administered to $\mathrm{BALB} / \mathrm{c}$ mice by tail vein injection. Twenty-four hours later, $15 \mathrm{mg} / \mathrm{ml}$ LPS was given by intraperitoneal injection to induce endotoxic shock. Three days later, Tim- 4 mRNA and protein expression in liver, spleen, lung, and kidney was determined. The survival rate of mice in the two groups was observed for $40 \mathrm{~h}$. The serum concentrations of tumor necrosis factor- $\alpha$ (TNF- $\alpha$ ) and interleukin-6 (IL-6) were measured using ELISA kits (eBioscience Biotec, San Diego, USA).

Knockdown of Tim- 4 was conducted. A mixture of mU6Tim-4-shRNA or mU6 plasmid DNA and PEI at 10:1 was administered to $\mathrm{BALB} / \mathrm{c}$ mice. Twenty-four hours later, $15 \mathrm{mg} / \mathrm{ml}$ LPS was given by intraperitoneal injection to induce endotoxic shock. Three days later, Tim- 4 mRNA and protein expression in spleen and lung, the survival rate and the concentration of TNF- $\alpha$ and IL- 6 were detected as described above.

\section{Macrophage Isolation}

Peritoneal macrophages were isolated from BALB/c mice. Briefly, $1 \mathrm{ml}$ of a sterile $6 \%$ starch solution was injected into the abdominal cavity for $72 \mathrm{~h}$. Serum-free DMEM medium was used to rinse the peritoneal lavage and the exudated cells were collected. The cells were cultured in DMEM with 10\% fetal bovine serum in 24-well plates and used for subsequent experiments.

\section{Transfection}

A total of either $0.2,0.6$, or $1 \mu \mathrm{g}$ pcDNA3-Tim-4 or mU6Tim-4-shRNA and their control plasmids (pcDNA3, mU6, respectively) were transfected into macrophages by Lipofectamine2000. Forty-eight hours later, $100 \mathrm{ng} / \mathrm{ml}$ of LPS was used to stimulate the macrophages for $24 \mathrm{~h}$. The concentrations of IL- 6 and TNF- $\alpha$ in supernatant fractions were detected by ELISA.

\section{Tim-4 Blockade}

Either $1,2.5$, or $5 \mu \mathrm{g} / \mathrm{ml}$ of Tim-4 antibody (Cat\# AF2826, R\&D, USA) or $10 \mu \mathrm{g} / \mathrm{ml}$ of control goat IgG (R\&D, USA) was incubated with macrophages for $1 \mathrm{~h}$, then LPS was used to stimulate for $24 \mathrm{~h}$ and IL-6, TNF- $\alpha$ levels in supernatant fractions were detected by ELISA.

\section{NF-KB Signal Inhibition}

Peritoneal macrophages or stable cell line RAW264.7-Tim-4 (cells overexpressing Tim-4) and control cells RAW264.7pcDNA3 were pretreated with $83.3 \mu \mathrm{g} / \mathrm{ml}$ of NF- $\kappa$ B inhibitory ligand (Cat\# 25-007, upstate, USA) for $30 \mathrm{~min}$, then incubated with $5 \mu \mathrm{g} / \mathrm{ml} \mathrm{Tim}-4$ blocking antibody or isotype control IgG for $1 \mathrm{~h}$. LPS (100 ng/ml) was used to stimulate for $24 \mathrm{~h}$, and TNF- $\alpha$ and IL-6 levels in supernatants were measured by ELISA.

\section{Semi-Quantitative RT-PCR and Quantitative Real-Time PCR}

Total cellular RNA was extracted using TRIzol reagent (Invitrogen, Carlsbad, CA, USA) and cDNA synthesized according to the manufacturer's instructions. Specific primers 
a

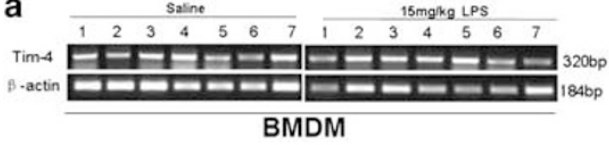

BMDM

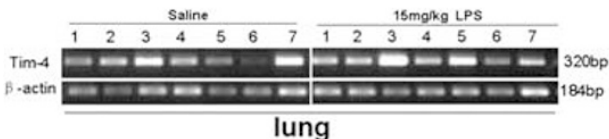

lung

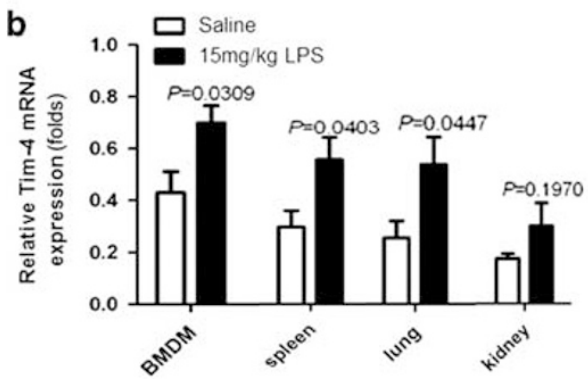

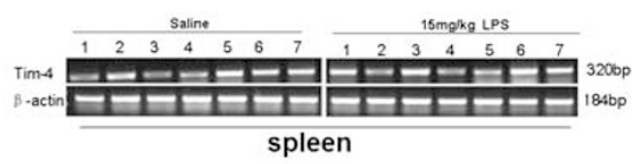
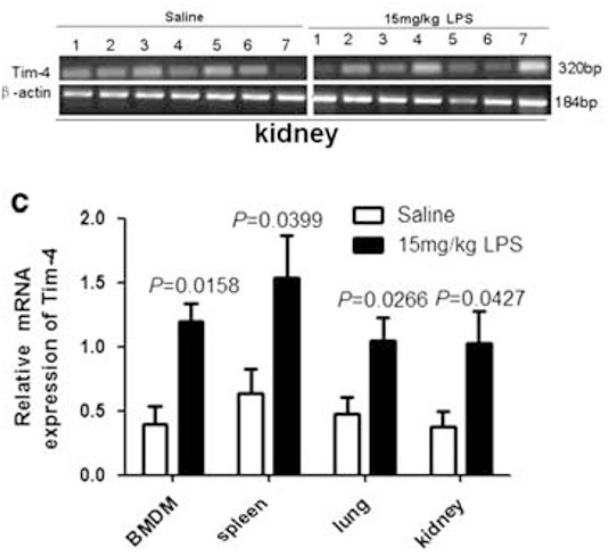

Figure 1 Tim-4 (T-cell immunoglobulin- and mucin-domain-containing molecule-4) expression is increased in lipopolysaccharide (LPS)-induced endotoxic shock. BALB/c mice were administered LPS (15 mg/kg) by intraperitoneal injection, and saline was used as control. Eight hours later, bone marrow, spleen, lung, and kidney were excised and RT-PCR was used to detect Tim-4 mRNA expression (a). The relative expression level of Tim-4 mRNA was analyzed by Image-J software and student's $t$-test (b). Real-time RT-PCR was also used to detect Tim-4 mRNA expression in these organs (c).

used for RT-PCR assays were as follows: 5'-CTACAGACA TAGCCGTACTCA-3' (sense) and 5'-GTCTTCATCATCCCT CCC-3' (antisense) for Tim-4, 5'-TGCGTGACATCAAA GAGAAG-3' (sense) and 5'-TCCATACCCAAGAAG-3' (antisense) for $\beta$-actin. Quantitative real-time PCR reaction was run on CFX96 Real-time PCR system (Bio-rad, USA) as follows: $95^{\circ} \mathrm{C}$ for $10 \mathrm{~min}$ (an initial denaturation step), followed by 40 cycles of $95^{\circ} \mathrm{C}$ for $15 \mathrm{~s}$ and $60^{\circ} \mathrm{C}$ for $1 \mathrm{~min}$ (annealing and extension). In addition, melting curve (55$95^{\circ} \mathrm{C}$ ) was performed at the end of each run. Gene-specific primers were as follows: Tim-4, forward (5'-ACAGGACAGA TGGATGGAATACCC- $3^{\prime}$ ) and reverse (5'-AGCCTTGTGTT TCTGCG-3'); $\beta$-actin, forward (5'-GGCATCGTGATGGA CTCCG-3') and reverse (5'-GCTGGAAGGTGGACAGCG A-3'). The relative expression of target gene was calculated considering its amplification efficiencies.

\section{Western Blotting}

Tissues were ground and lysed by Cell LyticäM (Sigma) and added to gel-loading buffer. After boiling for $10 \mathrm{~min}$, proteins were resolved by SDS-polyacrylamide gel electrophoresis and electro-blotted onto a polyvinylidene fluoride membrane (Millipore, Jinan, China). Membranes were blocked with 3\% bovine serum albumin and incubated with indicated antibodies. After washing in PBS-containing 0.5\% Tween 20, the bound primary antibody was detected with anti-rabbit IgG or anti-mouse IgG (Zhongshan, Beijing, China). After washing, the antibody-bound protein was visualized by enhanced chemiluminescence. Antibodies used are as follows: antiTim-4 (SAB3500444, Sigma), anti-GAPDH (60004-I-Ig, proteintech TM), and anti- $\beta$-Actin (TA-09, Zhongshanjinqiao).

\section{ELISA}

TNF- $\alpha$ and IL- 6 concentrations in culture supernatants or serum were measured by ELISA kits according to the manufacturer's instructions.

\section{Statistical Analysis}

Unpaired Student's t-test (GraphPad Prism 5.0; GraphPad Software, San Diego, CA, USA) was used for comparison between two groups. Survival plots of mice with treated group and control were constructed using the Kaplan-Meier method and were compared using the log rank test. A value of $P<0.05$ was considered significant.

\section{RESULTS}

\section{Tim-4 Expression is Increased in LPS-Induced Endotoxic Shock}

Previously, our group had shown that Tim-4 was significantly induced by ConA, interferon- $\gamma$ and LPS in macrophages in vitro. ${ }^{16,17}$ To examine whether Tim-4 is also induced by LPS in vivo, BALB/c mice were administered LPS by intraperitoneal injection and the expression level of Tim-4 mRNA was measured by RT-PCR and real-time RT-PCR. As shown in Figure 1, Tim-4 mRNA expression in bone marrow, spleen, lung, and kidney was significantly induced by LPS administration. Tim-4 protein expression was also enhanced in these organs after LPS stimulation (Supplementary Figure 1A). In addition, similar results were observed in C57BL/6 mice (Supplementary Figure 1B). These results suggest that LPS-induced Tim-4 may have a role in the regulation of the inflammatory response. 

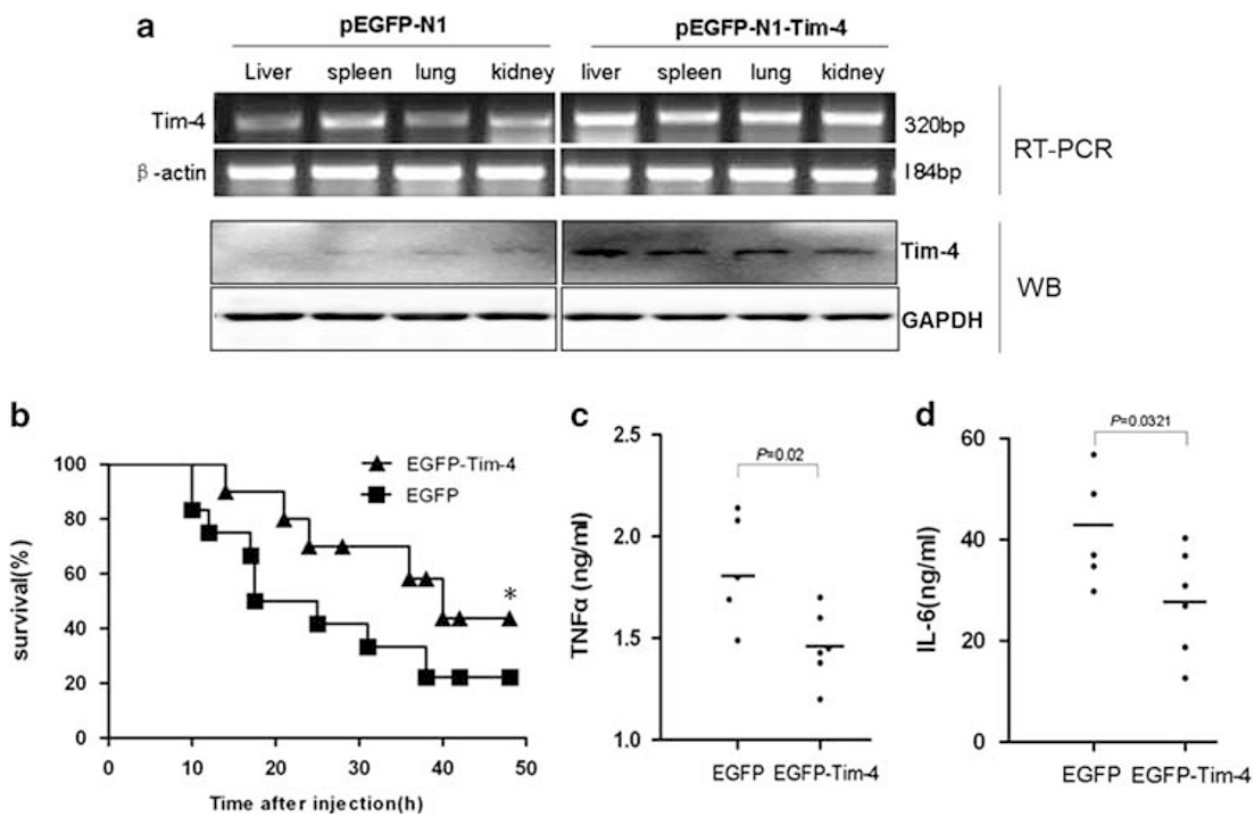

Figure 2 Tim-4 (T-cell immunoglobulin- and mucin-domain-containing molecule-4) overexpression attenuates lipopolysaccharide (LPS)-induced endotoxic shock. A mixture of $60 \mu \mathrm{g}$ pEGFP-N1-Tim-4 or pEGFP-N1 plasmid DNA and PEl at 10:1 was administered to BALB/c mice by tail vein injection. Three days later, the mice were sacrificed and Tim-4 mRNA and protein expression in liver, spleen, lung, and kidney was detected by RT-PCR or western blotting (a). Twenty-four hours after Tim-4 or control plasmid administration, $15 \mathrm{mg} / \mathrm{ml}$ of LPS was given by intraperitoneal injection to induce endotoxic shock. The survival rate of mice in the two groups was observed for $40 \mathrm{~h}(\mathbf{b})\left({ }^{*} P<0.05\right)$. Tumor necrosis factor- $a(\mathbf{c})$ and interleukin-6 (d) in serum was measured by ELISA.

\section{Tim-4 Attenuates LPS-Induced Endotoxic Shock}

To further investigate the function of Tim- 4 in inflammation induced by LPS, the Tim- 4 eukaryotic expression plasmid was administered to $\mathrm{BALB} / \mathrm{c}$ mice before LPS injection. Three days later, the mice were killed and Tim- 4 mRNA and protein expression in liver, spleen, lung, and kidney was detected by RT-PCR or western blotting. As shown in Figure 2, the expression of Tim-4 was increased in these organs in experimental group (a) as compared with the control group. Moreover, the survival rate of mice in the Tim- 4 overexpression group was $45 \%$ within $40 \mathrm{~h}$ after LPS treatment. However, the survival rate of the control group was only $23 \%$ (b). The concentrations of TNF- $\alpha$ (c) and IL-6 (d) in serum were lower in Tim-4 overexpression group compared with the controls as well.

To further confirm the role of Tim-4 in vivo, we constructed the Tim-4-shRNA plasmid to interfere with Tim-4 expression. As shown in Figure 3, the expression of Tim-4 in spleen and lung in Tim-4-shRNA group decreased correspondingly. The survival rate of Tim-4 knockdown mice was $18 \%$, whereas the control group was $32 \%, 40 \mathrm{~h}$ after LPS treatment (b). The concentrations of TNF- $\alpha$ (c) and IL-6 (d) in serum were higher in Tim-4-shRNA group compared with controls as well. Taken together, these results indicate that Tim-4 protects mice from LPS-induced septic shock.

\section{Tim-4 Inhibits Cytokine Production of Macrophages}

Macrophages are the primary source of cytokines such as IL-6 and TNF- $\alpha$ in the process of inflammation. To further investigate whether Tim- 4 protects mice from LPS-induced septic shock by inhibiting macrophages, we isolated the peritoneal macrophages from $\mathrm{BALB} / \mathrm{c}$ mice and transfected overexpression and knockdown plasmids of Tim-4 to macrophages before LPS treatment. Moreover, Tim- 4 antibody was incubated with macrophages for $1 \mathrm{~h}$ before LPS stimulation. The IL- 6 and TNF- $\alpha$ levels in supernatants were detected by ELISA. As shown in Figure 4, the concentrations of IL- 6 and TNF- $\alpha$ in Tim-4 overexpression group were decreased in contrast to control group. Consistently, the levels of inflammatory factors in Tim-4 knockdown or blocking group were increased in contrast to the controls. These results further verified the inhibitory effect of Tim-4 on the production of inflammatory cytokines in macrophages.

\section{Tim-4 Inhibits Cytokine Production via NF-KB Signaling Pathway}

$\mathrm{NF}-\kappa \mathrm{B}$ is one of the pivotal transcription factors regulating gene expression in inflammatory responses. Therefore, we speculated that Tim- 4 might participate in the process of LPSinduced endotoxic shock by inhibiting cytokine production. We constructed the stable cell line RAW264.7-Tim-4, which overexpressed Tim- 4 and pretreated them with NF- $\kappa \mathrm{B}$ inhibitory ligand for $30 \mathrm{~min}$ before LPS stimulation. As 

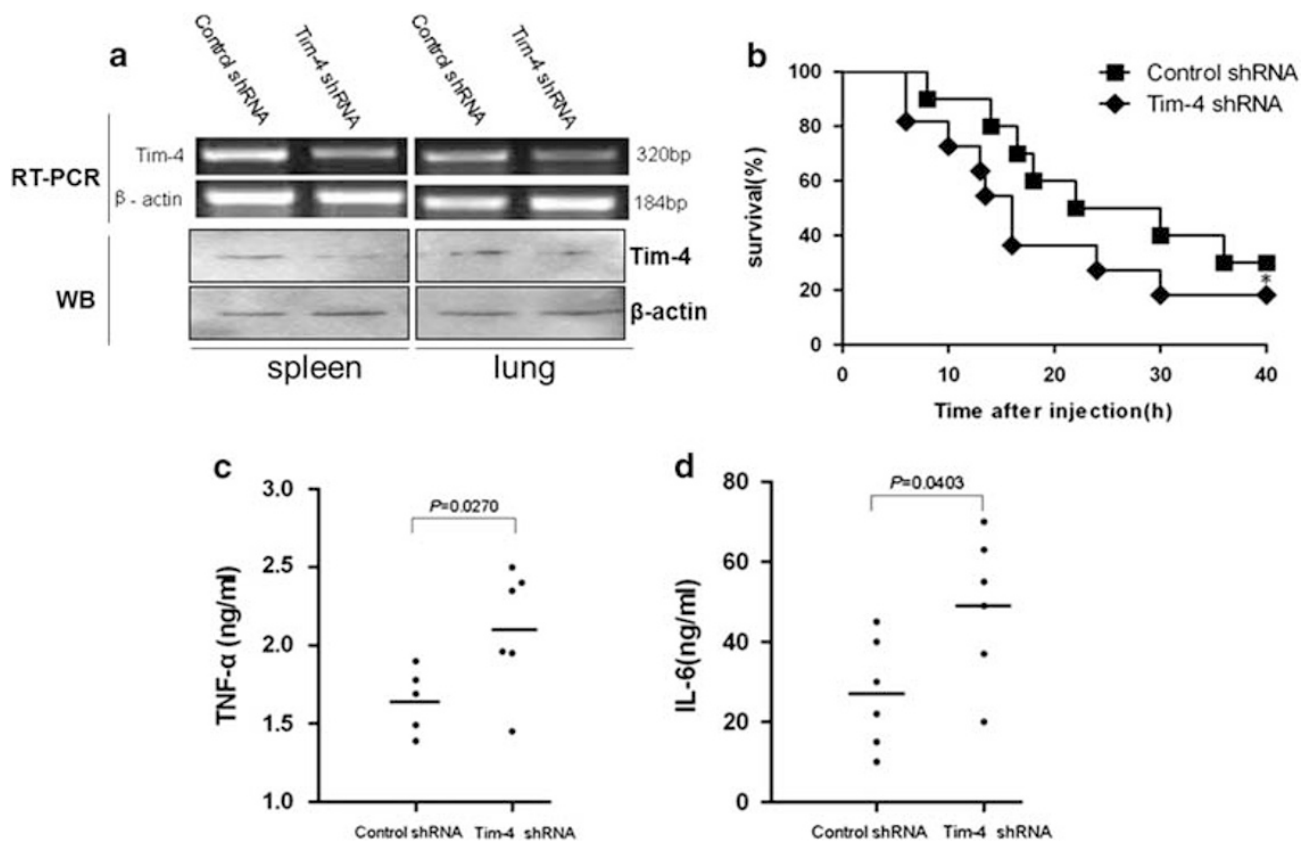

Figure 3 Tim-4 (T-cell immunoglobulin- and mucin-domain-containing molecule-4) knockdown aggravates lipopolysaccharide (LPS)-induced endotoxic shock. A mixture of $60 \mu \mathrm{g}$ mU6-Tim-4-shRNA or mU6 plasmid DNA and PEl at 10:1 was administered to BALB/c mice by tail vein injection. Three days later, the mice were sacrificed and Tim-4 mRNA and protein expression in spleen and lung was detected by RT-PCR or western blotting (a). Twenty-four hours after Tim-4-shRNA or control plasmid administration, $15 \mathrm{mg} / \mathrm{ml}$ of LPS was given by intraperitoneal injection to induce endotoxic shock. The survival rate of mice in the two groups was observed for $40 \mathrm{~h}(\mathbf{b})(* P<0.05)$. Tumor necrosis factor- $a(\mathbf{c})$ and interleukin- 6 (d) in serum was measured by ELISA.

shown in Figure 5, the TNF- $\alpha$ (a) and IL-6 (b) levels in supernatants from Tim- 4 overexpression macrophages were decreased compared with control; however, NF- $\kappa$ B inhibitory ligand reversed the decrease of TNF- $\alpha$ and IL- 6 levels. Peritoneal macrophages were also pretreated with $\mathrm{NF}-\kappa \mathrm{B}$ inhibitory ligand and then incubated with Tim- 4 blocking antibody before LPS treatment. The results show that TNF- $\alpha$ (c) and IL-6 (d) levels in supernatants of Tim-4 blocking group were increased compared with control. NF- $\kappa \mathrm{B}$ inhibitory ligand also abrogated the differences between two groups. These results suggest that Tim-4 may inhibit cytokine production via NF- $\kappa \mathrm{B}$ signaling pathway.

\section{DISCUSSION}

Stimulation of macrophages is essential in the development of endotoxic shock. ${ }^{19}$ Bacterial components, especially LPS, activate macrophages to produce proinflammatory cytokines, such as TNF- $\alpha$, IL-1, IL-6, and other inflammatory mediators, ${ }^{20}$ which then trigger a cascade of reactions that contributes to systemic inflammation. ${ }^{21}$ Previous studies by our group have shown that Tim-4 expression on HMCs increased in a mouse model after ConA administration, coincident with the increased expression of Tim- 4 on macrophages after stimulation. ${ }^{16,17}$ Moreover, we observed increased Tim-4 expression on PBMCs from systemic lupus erythematosus ${ }^{22}$ and type 2 diabetes patients. ${ }^{23}$ It therefore appears that the potential role of Tim- 4 is to downregulate the innate immune system, particularly macrophages, in order to decrease excessive inflammatory reactions. Recently, we identify Tim-4 as a critical regulator of LPS-induced macrophage activation by inhibiting $\mathrm{NO}$ production in vitro. ${ }^{17}$ These observations collectively support the hypothesis that Tim-4 has functional roles in attenuating inflammation-related diseases. In the current study, we further demonstrate that Tim- 4 protects mice against LPSinduced endotoxic shock by suppressing the activation of macrophages through the NF- $\kappa \mathrm{B}$ signaling pathway. These observations suggest that Tim-4 might be involved in the pathogenesis of macrophage-mediated endotoxic shock by inhibiting inflammatory factor production.

Normally, the inflammatory response in sepsis involves a complex interplay of different biological systems and cell types, resulting in severe dysregulation of the inflammatory network. ${ }^{24}$ At the onset of endotoxic shock, a nonresolving inflammatory reaction occurs, which can lead to organ damage and dysfunction. Subsequently, cytokines attract multiple immune cells, including macrophages, and these recruited cells produce even more cytokines to perpetuate the response. ${ }^{25}$ Excessive production of proinflammatory cytokines, especially TNF-a and IL-6, leading to endothelial damage, increased microvascular permeability, platelet aggregation, myocardial dysfunction and ischemia/reperfusion (I/R) injury, ${ }^{25,26}$ which frequently leads to organism disability and body death in endotoxic shock. Therefore, the 

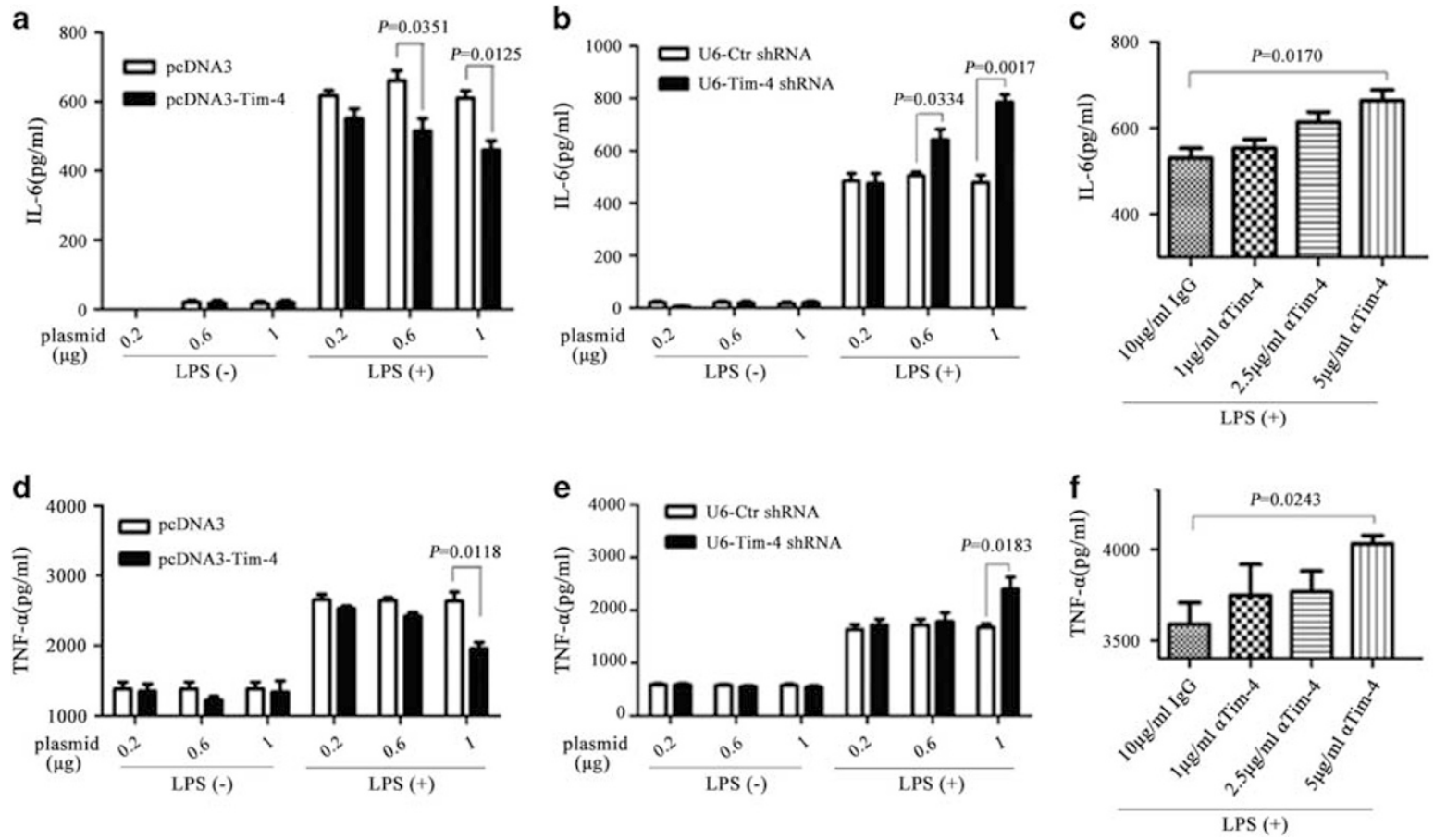

Figure 4 Tim-4 (T-cell immunoglobulin- and mucin-domain-containing molecule-4) inhibits cytokines production of macrophages. Peritoneal macrophages were isolated regularly from BALB/c mice. pcDNA3-Tim-4 plasmid DNA or pcDNA3 $(0.2,0.6$, and $1 \mu \mathrm{g})$ were transfected into macrophages by Lipofectamine2000. Forty-eight hours later, LPS (final concentration, $100 \mathrm{ng} / \mathrm{ml}$ ) was used to stimulate the macrophages for $24 \mathrm{~h}$, and interleukin-6 (IL-6) (a) and tumor necrosis factor- $a$ (TNF- $a$ ) (d) levels in supernatants were detected by ELISA. A total of 0.2, 0.6, and 1 $\mu$ g mU6-Tim-4-shRNA plasmid DNA or mU6 were transfected into macrophages by Lipofectamine2000. Forty-eight hours later, lipopolysaccharide (LPS; final concentration was $100 \mathrm{ng} /$ $\mathrm{ml}$ ) was used to stimulate the macrophages for $24 \mathrm{~h}$, and IL-6 (b) and TNF- $a$ (e) levels in supernatants were detected by ELISA. 1, 2.5 or $5 \mu \mathrm{g} / \mathrm{ml}$ of Tim4 antibody was incubated with macrophages for $1 \mathrm{~h}$, then $100 \mathrm{ng} / \mathrm{ml}$ of LPS was used to stimulate for $24 \mathrm{~h}$ and IL-6 (c), TNF- $a$ (f) levels were detected by ELISA.

uncontrolled inflammatory reaction induced by macrophagederived inflammatory cytokines is the primary mechanism in the process of sepsis. In our study, we demonstrated that Tim-4 expression was increased in LPS-induced experimental endotoxic shock, indicating that Tim- 4 might participate in the process. To further investigate the role of Tim- 4 in the development of endotoxic shock induced by LPS in vivo, Tim4 eukaryotic expression and interference plasmids were administered to BALB/c mice before LPS injection. The results show that Tim- 4 can attenuate the levels of inflammatory factors such as TNF-a and IL-6. More importantly, the survival rate of the Tim- 4 overexpression group was higher than controls. Accordingly, the survival rate of mice in the Tim- 4 downregulated group was lower. Tim-4 is exclusively expressed on macrophages and mature DCs, and regulates T-cell functions or mediates the phagocytosis of apoptotic cells through affecting the functions of macrophages. ${ }^{10,11,13-15,27,28}$ Combined with our previous data, these results indicate that Tim- 4 may suppress the degree of inflammation and protect mice from LPS-induced septic shock at least partially in vivo.

Macrophages are primary producer of TNF- $\alpha$ and IL-6, and TNF- $\alpha$ is the critical driver of septic shock. ${ }^{29}$ Studies have shown that Tim- 4 may modulate secretion of TNF- $\alpha$ by regulating the ability of resident peritoneal macrophages, ${ }^{27}$ which is consistent with our published data. ${ }^{16}$ To confirm whether Tim-4 participates in endotoxic shock by regulating the functions of macrophages, we included Tim- 4 overexpression and interference plasmids or blocking antibody to macrophages before LPS stimulation. The results show that TNF- $\alpha$ and IL- 6 levels in supernatants were decreased in the Tim- 4 overexpression group, and increased when interference plasmid or blocking antibody was given before LPS stimulation. Although Tim- 4 is also found on NKT and B1 cells, Tim- 4 is selectively highly expressed on macrophages and mature myeloid-derived dendritic cells. Therefore, we speculate that Tim-4 may suppress the degree of endotoxic shock at least partially by regulating the function of macrophages. In the future, the in vivo role of Tim-4 in LPS-induced endotoxic shock by regulating macrophages should be further identified using macrophage specific Tim-4 gene conditional knockout mice.

LPS is the major factor in the development of septic shock, ${ }^{30}$ and it initiates the inflammatory response through the TLR4 signaling pathway. ${ }^{31}$ In the process of endotoxic shock, LPS binds to LPS-binding protein and is delivered to 
a
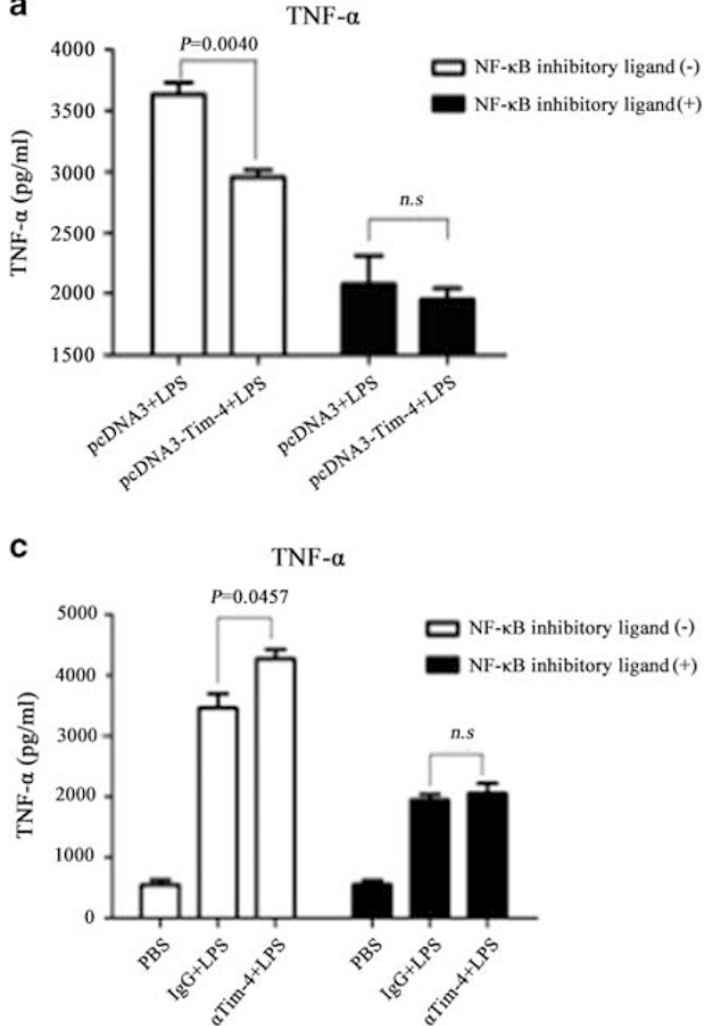

b

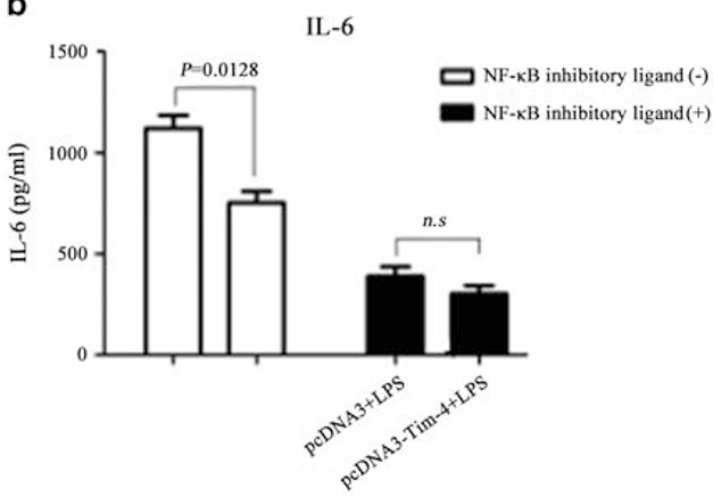

d

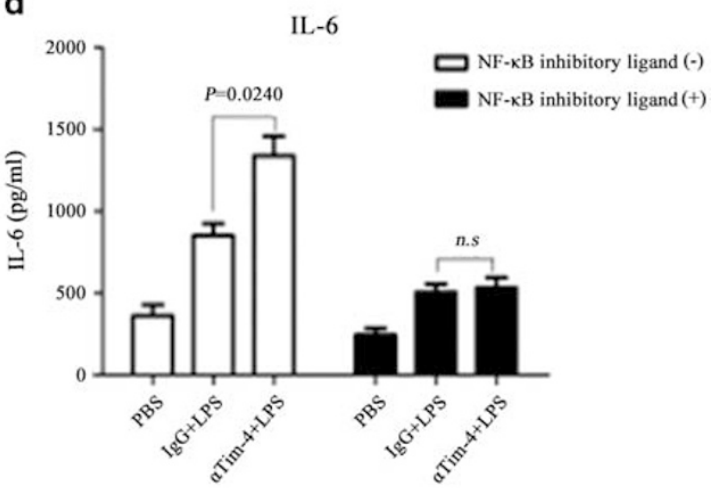

Figure 5 Tim-4 (T-cell immunoglobulin- and mucin-domain-containing molecule-4) inhibits cytokine production via nuclear factor-kappa B (NF- $K$ B) signaling pathway. Tim-4 overexpression stable cell line RAW264.7-Tim-4 and control cells RAW264.7-pcDNA3 were pretreated with 83.3 $\mu$ g/ml of NF-kB inhibitory ligand for $30 \mathrm{~min}$. Lipopolysaccharide (LPS; $100 \mathrm{ng} / \mathrm{ml}$ ) was used to stimulate these cells for $24 \mathrm{~h}$, and tumor necrosis factor- $a$ (TNF- $a$ ) (a) and interleukin-6 (IL-6) (b) levels in supernatants were measured by ELISA. Peritoneal macrophages were pretreated with $83.3 \mu \mathrm{g} / \mathrm{ml}$ of NF-kB inhibitory ligand for $30 \mathrm{~min}$, then incubated with $5 \mu \mathrm{g} / \mathrm{ml} \mathrm{Tim-4} \mathrm{blocking} \mathrm{antibody} \mathrm{or} \mathrm{isotype} \mathrm{control} \mathrm{lgG} \mathrm{for} 1 \mathrm{~h}$. LPS (100 ng/ml) was used to stimulate these cells for $24 \mathrm{~h}$, and TNF- $a$ (c) and IL-6 (d) levels in supernatants were measured by ELISA.

CD14 on the surface of macrophages, which leads to the interaction with the TLR4/myeloid differentiation protein-2 complex. $^{32}$ This interaction leads to activation of NF- $\kappa \mathrm{B}$ through the MyD88 dependent pathway, and ultimately results in the production of cytokines and other inflammatory molecules. ${ }^{33}$ In the development of endotoxic shock, undue cell stimulation might result in an uncontrolled host response leading to tissue damage and organ dysfunction. To clarify how Tim-4 works in the suppression of inflammatory factors by regulating macrophages, NF- $\kappa \mathrm{B}$ inhibitory ligand was administered before LPS stimulation. The NF- $\kappa \mathrm{B}$ transcription factor is a critical regulator of immunologically mediated immediate transcriptional responses, especially in LPSinduced macrophage activation and proinflammatory cytokine production. ${ }^{34}$ The results demonstrate that the levels of inflammatory factors such as TNF- $\alpha$ and IL-6 were decreased markedly, indicating that Tim-4 inhibits cytokine production via NF- $\mathrm{kB}$ signaling pathway, at least in part. We have revealed that Tim- 4 inhibits NO production and iNOS expression via NF-kB signaling pathway. ${ }^{17}$ It is reported that mice deficient in iNOS gene are more resistant to
LPS-induced acute lung injury than wild-type mice. ${ }^{35}$ Therefore, we speculate that Tim- 4 might protect mice from LPS-induced endotoxic shock partially by inhibiting NO production. In addition, Tim- 4 has been identified as a phosphatidylserine receptor that mediates the phagocytosis of apoptotic cells by macrophages ${ }^{13-15}$ and may be responsible for the maintenance of the homeostatic state of resident peritoneal macrophages. ${ }^{27}$ Though endothelial cell apoptosis is considered to be crucial for the development of severe sepsis/septic shock, ${ }^{36}$ uptake of apoptotic cells inhibits the proinflammatory mediators such as TNF- $\alpha$ and increases the immunosuppressive cytokine TGF- $\beta$ secreted by activated macrophages. Moreover, apoptotic cells protect mice against LPS-induced shock. ${ }^{37}$ It is reasonable that Tim- 4 might be involved in LPS-induced endotoxic shock by promoting the uptake of apoptotic cells.

Our previous studies have shown that the phosphorylation of NF- $\kappa \mathrm{B}$ p65 induced by LPS was inhibited in macrophages that overexpressed Tim-4, whereas its phosphorylation was promoted when Tim-4 was blocked. ${ }^{17}$ It is well known that the activation of NF- $\kappa \mathrm{B}$ is linked to a sequential cascade that 
IKK-dependent $\mathrm{I} \kappa \mathrm{B} \alpha$ degradation and the phosphorylation of $\mathrm{I} \kappa \mathrm{B} \alpha$ at serine residues, which is essential for the activation and release of NF- $\kappa$ B. ${ }^{38}$ Tim- 4 does not contain a tyrosinekinase phosphorylation motif in the cytoplasmic domain; ${ }^{10}$ therefore, the mechanisms of interaction between Tim-4 and NF- $\kappa \mathrm{B}$ pathway are not yet clear. A recent report has shown that Tim-4 can affect macrophage phagocytosis by interacting with integrin co-receptors. ${ }^{39}$ The adhesion receptor $\alpha \mathrm{V} \beta 3$ integrin ligation results in sustained increases of NF- $\kappa$ B DNA-binding activity in human blood monocytes and monocyte-derived macrophages. ${ }^{40}$ Tim- 4 contains an RGD motif in $\mathrm{IgV}$ domain and interacts with $\alpha \mathrm{V} \beta 3$ integrin in A549 cells. ${ }^{41}$ In addition, Tim-4 activates autophagy by directly interacting with AMPK $\alpha 1 .^{42}$ However, the exact mechanism of Tim- 4 downregulation of the NF- $\kappa$ B pathway in LPS-activated macrophages needs to be further investigated.

In summary, the present study demonstrates that Tim-4 attenuates LPS-induced endotoxic shock, and that Tim-4 inhibits cytokine production via macrophages in vitro. Additionally, we report that Tim-4 may suppress cytokine production via NF- $\kappa \mathrm{B}$ signaling pathway in the development of endotoxic shock, indicating Tim- 4 as a potent target for the treatment of endotoxic shock.

Supplementary Information accompanies the paper on the Laboratory Investigation website (http://www.laboratoryinvestigation.org)

\section{ACKNOWLEDGMENTS}

This work was supported in part by grants from the National Nature Science Foundation of China (No. 81172862, 81371831), Research Fund for the Doctoral Program of Higher Education of China (RFDP) (20110131110034), Key R \& D program in Shandong Province (No. 2015GSF118120) and the Shandong Provincial Natural Science Foundation (No. ZR2015HM031).

\section{DISCLOSURE/CONFLICT OF INTEREST}

The authors declare no conflict of interest.

1. Boomer JS, To K, Chang KC, et al. Immunosuppression in patients who die of sepsis and multiple organ failure. JAMA 2011;306: 2594-2605.

2. Singh $P$, Dejager $L, A m a n d ~ M$, et al. DUSP3 genetic deletion confers M2-like macrophage-dependent tolerance to septic shock. J Immunol 2015;194:4951-4962.

3. Janeway Jr. CA, Medzhitov R. Innate immune recognition. Annu Rev Immunol 2002;20:197-216.

4. Kumar $\mathrm{H}$, Kawai T, Akira S. Pathogen recognition by the innate immune system. Int Rev Immunol 2011;30:16-34.

5. Lopez-Bojorquez LN, Dehesa AZ, Reyes-Teran G. Molecular mechanisms involved in the pathogenesis of septic shock. Arch Med Res 2004;35:465-479.

6. Balk RA. Systemic inflammatory response syndrome (SIRS): where did it come from and is it still relevant today? Virulence 2014;5: 20-26.

7. King EG, Bauza GJ, Mella JR, et al. Pathophysiologic mechanisms in septic shock. Lab Invest 2014;94:4-12.

8. Angus DC, van der Poll T. Severe sepsis and septic shock. N Engl J Med 2013;369:840-851.

9. McIntire JJ, Umetsu SE, Akbari O, et al. Identification of Tapr (an airway hyperreactivity regulatory locus) and the linked Tim gene family. Nat Immunol 2001;2:1109-1116.
10. Meyers $\mathrm{JH}$, Chakravarti $\mathrm{S}$, Schlesinger $\mathrm{D}$, et al. TIM-4 is the ligand for TIM-1, and the TIM-1-TIM-4 interaction regulates $\mathrm{T}$ cell proliferation. Nat Immunol 2005;6:455-464.

11. Rodriguez-Manzanet $\mathrm{R}$, Meyers $\mathrm{JH}$, Balasubramanian S, et al. TIM-4 expressed on APCs induces T cell expansion and survival. J Immunol 2008;180:4706-4713.

12. Rodriguez-Manzanet R, DeKruyff R, Kuchroo VK, et al. The costimulatory role of TIM molecules. Immunol Rev 2009;229:259-270.

13. Kobayashi N, Karisola P, Pena-Cruz V, et al. TIM-1 and TIM-4 glycoproteins bind phosphatidylserine and mediate uptake of apoptotic cells. Immunity 2007;27:927-940.

14. Miyanishi $M$, Tada K, Koike $M$, et al. Identification of Tim4 as a phosphatidylserine receptor. Nature 2007;450:435-439.

15. Santiago C, Ballesteros A, Martinez-Munoz L, et al. Structures of T cell immunoglobulin mucin protein 4 show a metal-lon-dependent ligand binding site where phosphatidylserine binds. Immunity 2007;27: 941-951.

16. Xu L, Qi J, Zhao P, et al. T cell immunoglobulin- and mucin-domaincontaining molecule- 4 attenuates concanavalin A-induced hepatitis by regulating macrophage. J Leukoc Biol 2010;88:329-336.

17. Xu L, Qi J, Liu X, et al. Tim-4 inhibits NO generation by murine macrophages. PLoS One 2015;10:e0124771.

18. Mizui M, Shikina T, Arase $H$, et al. Bimodal regulation of $T$ cell-mediated immune responses by TIM-4. Int Immunol 2008;20:695-708.

19. Nathan C. Points of control in inflammation. Nature 2002;420:846-852.

20. Broad A, Jones DE, Kirby JA. Toll-like receptor (TLR) response tolerance: a key physiological "damage limitation" effect and an important potential opportunity for therapy. Curr Med Chem 2006;13:2487-2502.

21. Cohen J, Vincent JL, Adhikari NK, et al. Sepsis: a roadmap for future research. Lancet Infect Dis 2015;15:581-614.

22. Zhao $\mathrm{P}, \mathrm{Xu} \mathrm{L}$, Wang $\mathrm{P}$, et al. Increased expression of human T-cell immunoglobulin- and mucin-domain-containing molecule- 4 in peripheral blood mononuclear cells from patients with system lupus erythematosus. Cell Mol Immunol 2010;7:152-156.

23. Zhao $\mathrm{P}$, Wang $\mathrm{H}$, Li T, et al. Increased T cell immunoglobulin and mucin domain containing 4 (TIM-4) is negatively correlated with serum concentrations of interleukin-1beta in type 2 diabetes. J Diabetes 2015;8:199-205.

24. Rittirsch D, Flierl MA, Ward PA. Harmful molecular mechanisms in sepsis. Nat Rev Immunol 2008;8:776-787.

25. Zhao T, Li Y, Liu B, et al. Novel pharmacologic treatment attenuates septic shock and improves long-term survival. Surgery 2013;154: 206-213.

26. Gamkrelidze M, Intskirveli N, Vardosanidze K, et al. Myocardial dysfunction during septic shock (review). Georgian Med News 2014; 40-46.

27. Wong K, Valdez PA, Tan C, et al. Phosphatidylserine receptor Tim-4 is essential for the maintenance of the homeostatic state of resident peritoneal macrophages. Proc Natl Acad Sci USA 2010;107:8712-8717.

28. Mazaheri F, Breus O, Durdu S, et al. Distinct roles for BAl1 and TIM-4 in the engulfment of dying neurons by microglia. Nat Commun 2014;5: 4046.

29. Huang S, Miao R, Zhou Z, et al. MCPIP1 negatively regulates toll-like receptor 4 signaling and protects mice from LPS-induced septic shock. Cell Signal 2013;25:1228-1234.

30. Giacometti A, Cirioni O, Ghiselli $\mathrm{R}$, et al. Interaction of antimicrobial peptide temporin $\mathrm{L}$ with lipopolysaccharide in vitro and in experimental rat models of septic shock caused by gram-negative bacteria. Antimicrob Agents Chemother 2006;50:2478-2486.

31. Hansen FC, Kalle-Brune M, van der Plas MJ, et al. The thrombin-derived host defense peptide GKY25 inhibits endotoxin-induced responses through interactions with lipopolysaccharide and macrophages/ monocytes. J Immunol 2015;194:5397-5406.

32. Kim HM, Park BS, Kim Jl, et al. Crystal structure of the TLR4-MD-2 complex with bound endotoxin antagonist Eritoran. Cell 2007;130: 906-917.

33. Takeda K, Akira S. TLR signaling pathways. Semin Immunol 2004;16: 3-9.

34. Perkins ND. The Rel/NF-kappa B family: friend and foe. Trends Biochem Sci 2000;25:434-440.

35. Kristof AS, Goldberg P, Laubach V, et al. Role of inducible nitric oxide synthase in endotoxin-induced acute lung injury. Am J Respir Crit Care Med 1998;158:1883-1889. 
36. Fujita M, Kuwano K, Kunitake $\mathrm{R}$, et al. Endothelial cell apoptosis in lipopolysaccharide-induced lung injury in mice. Int Arch Allergy Immunol 1998;117:202-208.

37. Ren $Y$, Xie $Y$, Jiang $G$, et al. Apoptotic cells protect mice against lipopolysaccharide-induced shock. J Immunol 2008;180:4978-4985.

38. Lawrence T. The nuclear factor NF-kappaB pathway in inflammation. Cold Spring Harb Perspect Biol 2009;1:a001651.

39. Flannagan RS, Canton J, Furuya W, et al. The phosphatidylserine receptor TIM4 utilizes integrins as coreceptors to effect phagocytosis. Mol Biol Cell 2014;25:1511-1522.
40. Antonov AS, Antonova GN, Munn $\mathrm{DH}$, et al. alphaVbeta3 integrin regulates macrophage inflammatory responses via $\mathrm{PI} 3$ kinase/Aktdependent NF-kappaB activation. J Cell Physiol 2011;226:469-476.

41. Zhang $\mathrm{Q}$, Wang $\mathrm{H}, \mathrm{Wu} \mathrm{X}$, et al. TIM-4 promotes the growth of nonsmall-cell lung cancer in a RGD motif-dependent manner. Br J Cancer 2015;113:1484-1492.

42. Baghdadi M, Yoneda A, Yamashina T, et al. TIM-4 glycoproteinmediated degradation of dying tumor cells by autophagy leads to reduced antigen presentation and increased immune tolerance. Immunity 2013;39:1070-1081. 